\title{
Heliotrope Erkrankungen
}

\section{Eine und nur eine Antwort ist jeweils richtig!}

Welche Aussage ist richtig? A ... verminderte Pigmentierung der betroffenen Patienten. Primäre Photodermatosen $\quad B \quad$... immer einen bekannten Photosensibilisator. haben als wichtigsten pa- $\quad$ C ... elektromagnetische Strahlung als Auslöser. thogenetischen Faktor... $\quad$ D ... eine Enzymstörung.

E ... verminderte DNS-Reparaturkapazität.

Welche Aussage ist richtig? A ... enthält vor allem UV-Strahlung.

Das Sonnenspektrum ... $\quad$ B ... enthält vor allem Wärmestrahlung.

C ... enthält lediglich zu etwa $10 \%$ UV-Strahlung.

D ... enthält keinerlei Strahlung, die Infrarot genannt wird.

E ... hat für die Auslösung von Photodermatosen keine Bedeutung.

3 Welche Aussage ist richtig? Phototestverfahren...

A ... dienen der Überprüfung von UV-Geräten.

B ... sind für die Diagnostik von heliotropen Erkrankungen besonders bedeutsam.

C ... spielen lediglich wissenschaftlich eine Rolle.

D ... können bei Therapieentscheidungen keine Hilfe sein.

E ... folgen bei allen Lichtdermatosen einem einheitlichen Schema.

Welche Aussage ist richtig?
Die häufigste primäre Lichtdermatose ist...

A ... die Lichturtikaria.

B ... die Photoallergie.

C ... die polymorphe Lichtdermatose.

D ... das aktinische Retikuloid.

E ... der Lupus erythematodes.

Welche Aussage ist richtig?
Sekundäre Photodermatosen ...

A ... sind häufig internistische oder rheumatologische Erkrankungen, die durch Sonnenstrahlung verschlimmert werden können.

B ... haben als entscheidenden pathogenetischen Faktor die UV-Strahlung.

C ... haben immer eine gehäufte Hautkrebsrate.

D ... betreffen nur Menschen mit hellem Hauttyp.

E ... kommen in südlichen Ländern seltener vor. 
Welche Aussage ist richtig? A ... ist eine primäre Lichtdermatose.

Der Lupus erythematodes B ... ist eine heterogene Autoimmunerkrankung, die durch Sonnenstrahlung induziert $\cdots$ oder verschlimmert werden kann.

C ... wird durch UV-Strahlung zur Abheilung gebracht.

D ... zwingt die Patienten, ihren Tag/Nacht/Rhythmus zu wechseln.

E ... hat keine Beziehung zu Umweltfaktoren.

Welche Aussage ist richtig? A ... hat keine Assoziation mit Autoantikörpern.

Der subakute kutane Lupus B ... trägt keinerlei Risiko, in einen systemischen LE überzugehen. erythematodes...

C ... ist eine besonders lichtempfindliche Variante des LE-Spektrums.

D ... führt immer zur narbigen Alopezie.

E ... ist vergesellschaftet mit einer erhöhten Hautkrebsrate.

Welche Aussage zur Photoallergie ist richtig? Die Photoallergie...

A ... ist seltener als die phototoxische Dermatitis und kann durch systemisch eingenommene Medikamente oder extern applizierte Photosensibilisatoren ausgelöst werden.

B ... benötigt zur Auslösung keine UV-Strahlung.

C ... kann durch UV-Strahlung alleine ausgelöst werden.

D Der belichtete Epikutantest spielt bei der Diagnostik der Photoallergie keine Rolle.

E Das wichtigste diagnostische Verfahren zur Abklärung der Photoallergie ist die Intrakutantestung.

Welche Aussage ist richtig? A ... ist sehr selten.

Die polymorphe Lichtder- $\quad$ B ... kommt in Afrika und Südamerika am häufigsten vor. matose (PLD) ...

B ... kommt in Afrika und Südamerika am häufigsten vor.

D ... betrifft in Mitteleuropa mehr Frauen als Männer und hat hier eine Prävalenz von 20\%.

E ... wird durch einen bekannten exogenen Photosensibilisator vermittelt.

Welche Aussage ist richtig? Die chronisch aktinische ... ist eine Präkanzerose.

Dermatitis (CAD) ...

B ... ist gekennzeichnet durch Vorkommen von UV-induzierten Karzinomen.

C ... betrifft vor allem jüngere Patienten.

D ... ist eine stark juckende Dermatose, die durch chronisch erhöhte Lichtempfindlichkeit mit Ekzemen an UV-exponierten Partien gekennzeichnet ist.

E ... geht häufig mit einem Lupus erythematodes einher.

Welche Aussage zur Therapie der polymorphen Lichtdermatose (PLD) ist richtig? Die Therapie der PLD...

B ... muss immer systemisch erfolgen.

C ... besteht immer nur in der Verordnung von Lichtschutzmitteln.

D Zur Therapie der PLD muss eine Phototherapie eingeleitet werden.

E ... bedingt eine absolute Karenz der Sonnenexposition.

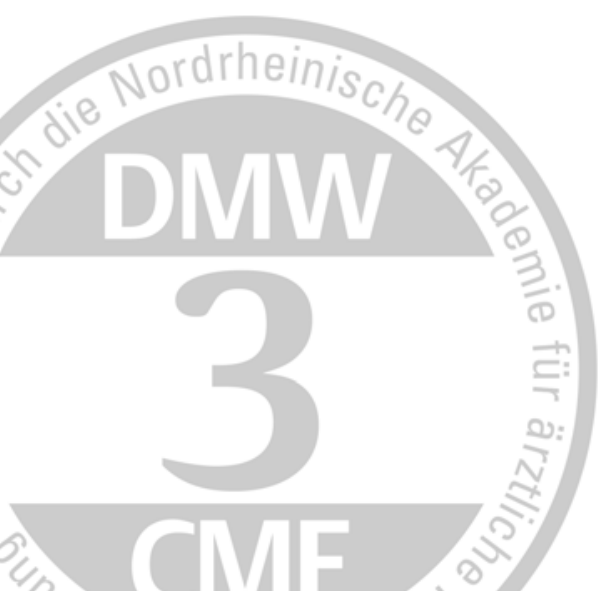

\section{Teilnahmebedingungen}

Für diese Fortbildungseinheit erhalten Sie drei Fortbildungspunkte im Rahmen des freiwilligen Fortbildungszertifikates. Hierfür

- müssen 10 der 11 Quiz-Fragen richtig beantwortet sein (bestanden hat auch, wer nicht weniger Fragen richtig beantwortet hat als der Durchschnitt aller Teilnehmer).

- müssen Seite 1 und Seite 2 des Evaluationsbogens vollständig ausgefüllt sein. Unvollständig ausgefültte Bögen können nicht berücksichtigt werden!

- muss eine CME-Wertmarke im Feld D (Evaluationsbogen) aufgeklebt oder Ihre DMW-Abonnement-Nummer eingetragen sein.

\section{Datenschutz}

Ihre Daten werden ausschließlich für die Bearbeitung dieser Fortbildungseinheit verwendet. Es erfolgt keine Speicherung der Ergebnisse über die für die Bearbeitung der Fortbildungseinheit notwendige Zeit hinaus. Die Daten werden nach Versand der Testate anonymisiert. Namens- und Adressangaben dienen nur dem Versand der Testate. Die Angaben zur Person dienen nur statistischen Zwecken und werden von den Adressangaben anonymisiert verarbeitet.

\section{Einsendeschluss ist der 6.4.2004}

(Datum des Poststempels). Die Zertifikate werden ab dem 14.4.2004 auf dem Postweg versandt. Von telefonischen Anfragen bitten wir abzusehen. Die richtige Auflösung des Quiz wird in der DMW Nr. 16/2004 (erscheint am 16.4.2004) veröffentlicht. 